Network Working Group

L. Masinter

Request for Comments: 2388

Category: Standards Track

\title{
Returning Values from Forms: multipart/form-data
}

Status of this Memo

This document specifies an Internet standards track protocol for the Internet community, and requests discussion and suggestions for improvements. Please refer to the current edition of the "Internet Official Protocol Standards" (STD 1) for the standardization state and status of this protocol. Distribution of this memo is unlimited.

Copyright Notice

Copyright (C) The Internet Society (1998). All Rights Reserved.

1. Abstract

This specification defines an Internet Media Type, multipart/formdata, which can be used by a wide variety of applications and transported by a wide variety of protocols as a way of returning a set of values as the result of a user filling out a form.

2. Introduction

In many applications, it is possible for a user to be presented with a form. The user will fill out the form, including information that is typed, generated by user input, or included from files that the user has selected. When the form is filled out, the data from the form is sent from the user to the receiving application.

The definition of Multipart/Form-Data is derived from one of those applications, originally set out in [RFC1867] and subsequently incorporated into [HTML40], where forms are expressed in HTML, and in which the form values are sent via HTTP or electronic mail. This representation is widely implemented in numerous web browsers and web servers.

However, multipart/form-data can be used for forms that are presented using representations other than HTML (spreadsheets, Portable Document Format, etc), and for transport using other means than electronic mail or HTTP. This document defines the representation of form values independently of the application for which it is used. 
3. Definition of multipart/form-data

The media-type multipart/form-data follows the rules of all multipart MIME data streams as outlined in [RFC 2046]. In forms, there are a series of fields to be supplied by the user who fills out the form. Each field has a name. Within a given form, the names are unique.

"multipart/form-data" contains a series of parts. Each part is expected to contain a content-disposition header [RFC 2183] where the disposition type is "form-data", and where the disposition contains an (additional) parameter of "name", where the value of that parameter is the original field name in the form. For example, a part might contain a header:

Content-Disposition: form-data; name="user"

with the value corresponding to the entry of the "user" field.

Field names originally in non-ASCII character sets may be encoded within the value of the "name" parameter using the standard method described in RFC 2047.

As with all multipart MIME types, each part has an optional "Content-Type", which defaults to text/plain. If the contents of a file are returned via filling out a form, then the file input is identified as the appropriate media type, if known, or "application/octet-stream". If multiple files are to be returned as the result of a single form entry, they should be represented as a "multipart/mixed" part embedded within the "multipart/form-data".

Each part may be encoded and the "content-transfer-encoding" header supplied if the value of that part does not conform to the default encoding.

4. Use of multipart/form-data

\subsection{Boundary}

As with other multipart types, a boundary is selected that does not occur in any of the data. Each field of the form is sent, in the order defined by the sending appliction and form, as a part of the multipart stream. Each part identifies the INPUT name within the original form. Each part should be labelled with an appropriate content-type if the media type is known (e.g., inferred from the file extension or operating system typing information) or as

"application/octet-stream". 


\subsection{Sets of files}

If the value of a form field is a set of files rather than a single file, that value can be transferred together using the

"multipart/mixed" format.

\subsection{Encoding}

While the HTTP protocol can transport arbitrary binary data, the default for mail transport is the 7BIT encoding. The value supplied for a part may need to be encoded and the "content-transfer-encoding" header supplied if the value does not conform to the default encoding. [See section 5 of RFC 2046 for more details.]

\subsection{Other attributes}

Forms may request file inputs from the user; the form software may include the file name and other file attributes, as specified in [RFC 2184 ] .

The original local file name may be supplied as well, either as a "filename" parameter either of the "content-disposition: form-data" header or, in the case of multiple files, in a "content-disposition: file" header of the subpart. The sending application MAY supply a file name; if the file name of the sender's operating system is not in US-ASCII, the file name might be approximated, or encoded using the method of RFC 2231.

This is a convenience for those cases where the files supplied by the form might contain references to each other, e.g., a TeX file and its sty auxiliary style description.

\subsection{Charset of text in form data}

Each part of a multipart/form-data is supposed to have a contenttype. In the case where a field element is text, the charset parameter for the text indicates the character encoding used.

For example, a form with a text field in which a user typed 'Joe owes <eu>100' where <eu> is the Euro symbol might have form data returned as:

$--\mathrm{AaB} 03 \mathrm{x}$

content-disposition: form-data; name="field1"

content-type: text/plain; charset=windows -1250

content-transfer-encoding: quoted-printable 
Joe owes $=80100$.

$--\mathrm{AaB} 03 \mathrm{x}$

5. Operability considerations

5.1 Compression, encryption

Some of the data in forms may be compressed or encrypted, using other MIME mechanisms. This is a function of the application that is generating the form-data.

5.2 Other data encodings rather than multipart

Various people have suggested using new mime top-level type "aggregate", e.g., aggregate/mixed or a content-transfer-encoding of "packet" to express indeterminate-length binary data, rather than relying on the multipart-style boundaries. While this would be useful, the "multipart" mechanisms are well established, simple to implement on both the sending client and receiving server, and as efficient as other methods of dealing with multiple combinations of binary data.

The multipart/form-data encoding has a high overhead and performance impact if there are many fields with short values. However, in practice, for the forms in use, for example, in HTML, the average overhead is not significant.

\subsection{Remote files with third-party transfer}

In some scenarios, the user operating the form software might want to specify a URL for remote data rather than a local file. In this case, is there a way to allow the browser to send to the client a pointer to the external data rather than the entire contents? This capability could be implemented, for example, by having the client send to the server data of type "message/external-body" with "access-type" set to, say, "uri", and the URL of the remote data in the body of the message.

\section{4 Non-ASCII field names}

Note that MIME headers are generally required to consist only of 7bit data in the US-ASCII character set. Hence field names should be encoded according to the method in RFC 2047 if they contain characters outside of that set. 
5.5 Ordered fields and duplicated field names

The relationship of the ordering of fields within a form and the ordering of returned values within "multipart/form-data" is not defined by this specification, nor is the handling of the case where a form has multiple fields with the same name. While HTML-based forms may send back results in the order received, and intermediaries should not reorder the results, there are some systems which might not define a natural order for form fields.

5.6 Interoperability with web applications

Many web applications use the "application/x-url-encoded" method for returning data from forms. This format is quite compact, e.g.:

name=Xavier+Xantico\&verdict $=$ Yes\&colour=Blue\&happy=sad\&Utf\%F $6 r=$ Send

however, there is no opportunity to label the enclosed data with content type, apply a charset, or use other encoding mechanisms.

Many form-interpreting programs (primarly web browsers) now implement and generate multipart/form-data, but an existing application might need to optionally support both the application/x-url-encoded format as well.

\subsection{Correlating form data with the original form}

This specification provides no specific mechanism by which multipart/form-data can be associated with the form that caused it to be transmitted. This separation is intentional; many different forms might be used for transmitting the same data. In practice, applications may supply a specific form processing resource (in HTML, the ACTION attribute in a FORM tag) for each different form. Alternatively, data about the form might be encoded in a "hidden field" (a field which is part of the form but which has a fixed value to be transmitted back to the form-data processor.)

6. Security Considerations

The data format described in this document introduces no new security considerations outside of those introduced by the protocols that use it and of the component elements. It is important when interpreting content-disposition to not overwrite files in the recipients address space inadvertently.

User applications that request form information from users must be careful not to cause a user to send information to the requestor or a third party unwillingly or unwittingly. For example, a form might 


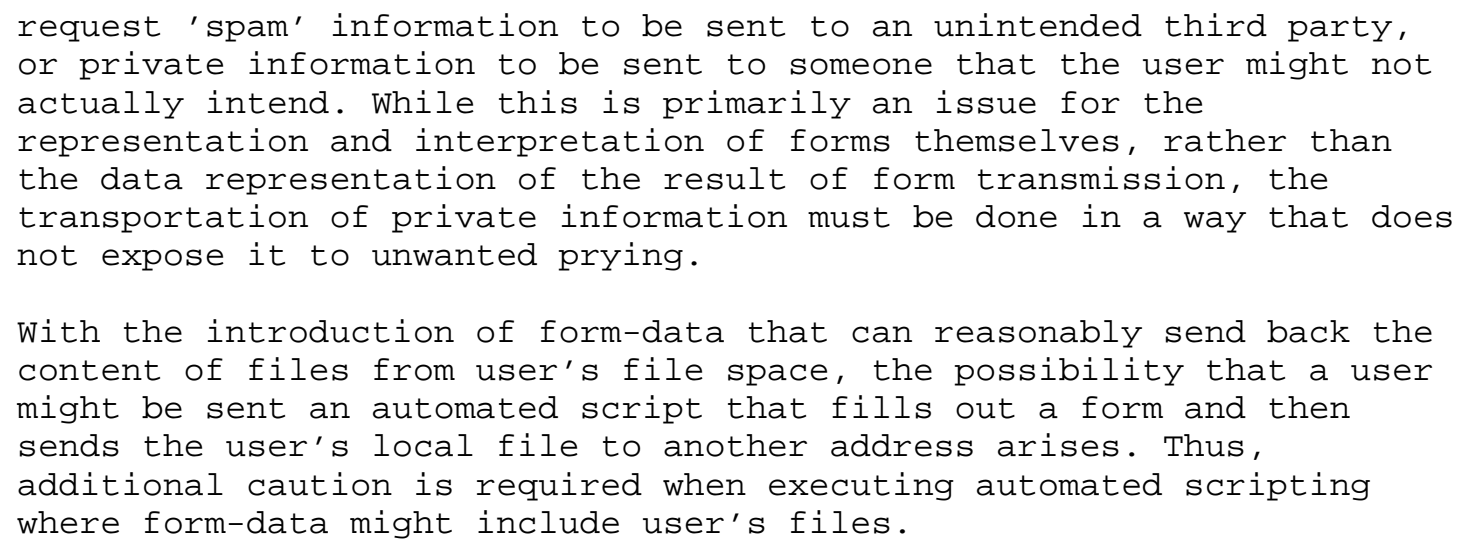

7. Author's Address

Larry Masinter

Xerox Palo Alto Research Center

3333 Coyote Hill Road

Palo Alto, CA 94304

Fax: $\quad+16508124333$

EMail: masinter@parc.xerox.com 


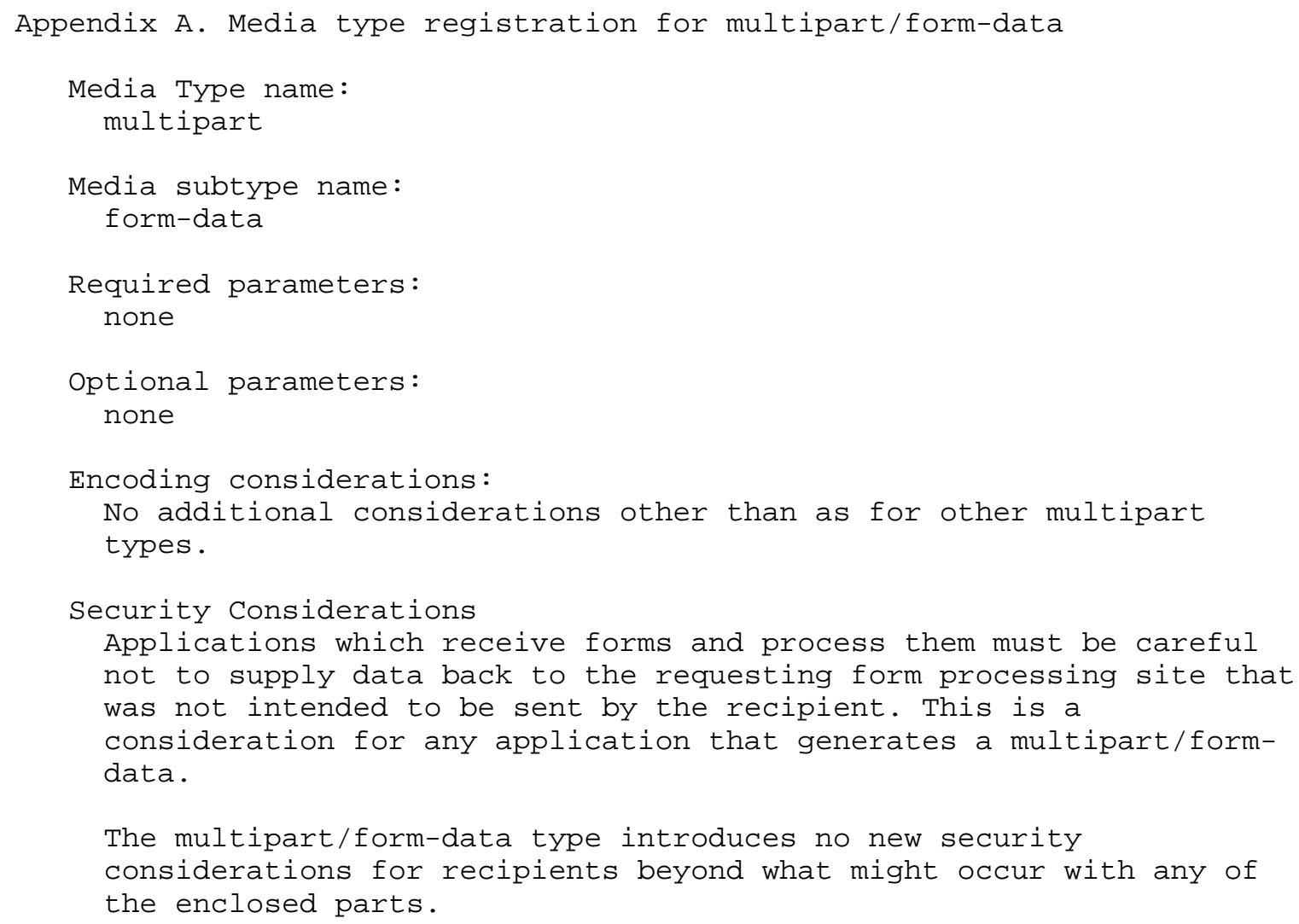


References

[RFC 2046] Freed, N., and N. Borenstein, "Multipurpose Internet Mail Extensions (MIME) Part Two: Media Types", RFC 2046, November 1996.

[RFC 2047] Moore, K., "MIME (Multipurpose Internet Mail Extensions) Part Three: Message Header Extensions for Non-ASCII Text", RFC 2047, November 1996.

[RFC 2231] Freed, N., and K. Moore, "MIME Parameter Value and Encoded Word Extensions: Character Sets, Languages, and Continuations", RFC 2231, November 1997.

[RFC 1806] Troost, R., and S. Dorner, "Communicating Presentation Information in Internet Messages: The Content-Disposition Header", RFC 1806, June 1995.

[RFC 1867] Nebel, E., and L. Masinter, "Form-based File Upload in HTML", RFC 1867, November 1995.

[RFC 2183] Troost, R., Dorner, S., and K. Moore, "Communicating Presentation Information in Internet Messages: The Content-Disposition Header Field", RFC 2183, August 1997.

[RFC 2184] Freed, N., and K. Moore, "MIME Parameter Value and Encoded Word Extensions: Character Sets, Languages, and Continuations", RFC 2184, August 1997.

[HTML40] D. Raggett, A. Le Hors, I. Jacobs. "HTML 4.0 Specification", World Wide Web Consortium Technical Report "REC-html40", December, 1997. <http://wWw.w3.org/TR/REChtml $40 />$ 
Full Copyright statement

Copyright (C) The Internet Society (1998). All Rights Reserved.

This document and translations of it may be copied and furnished to others, and derivative works that comment on or otherwise explain it or assist in its implementation may be prepared, copied, published and distributed, in whole or in part, without restriction of any kind, provided that the above copyright notice and this paragraph are included on all such copies and derivative works. However, this document itself may not be modified in any way, such as by removing the copyright notice or references to the Internet society or other Internet organizations, except as needed for the purpose of developing Internet standards in which case the procedures for copyrights defined in the Internet Standards process must be followed, or as required to translate it into languages other than English.

The limited permissions granted above are perpetual and will not be revoked by the Internet society or its successors or assigns.

This document and the information contained herein is provided on an "AS IS" basis and THE INTERNET SOCIETY AND THE INTERNET ENGINEERING TASK FORCE DISCLAIMS ALL WARRANTIES, EXPRESS OR IMPLIED, INCLUDING BUT NOT LIMITED TO ANY WARRANTY THAT THE USE OF THE INFORMATION HEREIN WILL NOT INFRINGE ANY RIGHTS OR ANY IMPLIED WARRANTIES OF MERCHANTABILITY OR FITNESS FOR A PARTICULAR PURPOSE. 